\title{
Measurements of forward energy flow and forward jet production with CMS
}

\author{
Hannes JUNG*i \\ CERN, Geneva, Switzerland \\ DESY, Hamburg, Germany \\ University Antwerp, Antwerp, Belgium \\ E-mail: hannes.jung@cern. de
}

\begin{abstract}
Measurements of the forward energy flow in minimum bias events and events with a high $p_{t}$ dijet system or a $\mathrm{W}$ boson in the central region are presented and compared to MC predictions which include a simulation of multiparton interactions. The measurements are not well described by the MC simulations.

Measurements of the forward jet cross section and the cross section associated with a central jet are presented and compared to theoretical predictions.
\end{abstract}

The 2011 Europhysics Conference on High Energy Physics, EPS-HEP 2011,

July 21-27, 2011

Grenoble, Rhône-Alpes, France

\footnotetext{
*Speaker.

†n behalf of the CMS collaboration
} 


\section{Energy Flow Measurements}

The energy flow in the forward region is directly sensitive to the amount of parton radiation and multiparton interaction. The energy flow is measured in the forward region of pseudorapidity $3.15<|\eta|<4.9$ with the CMS detector [1] for minimum bias events and events containing a dijet system in the central region $(|\eta|<2$. The measurements [2], corrected for detector effects, are shown in Fig. 1. The statistcal uncertainties are negligible, the systematic uncertainties, indicated with the error bars in the figure, are 11-14\% (13-22\%) for minimum bias (dijets) events. The uncertainty of the energy scale is $10 \%$. The model uncertainty of the correction from detecor to stable particle level is 1-3\% (12-17\%) for minimum bias (dijet) events. In Fig. 1a the mea-
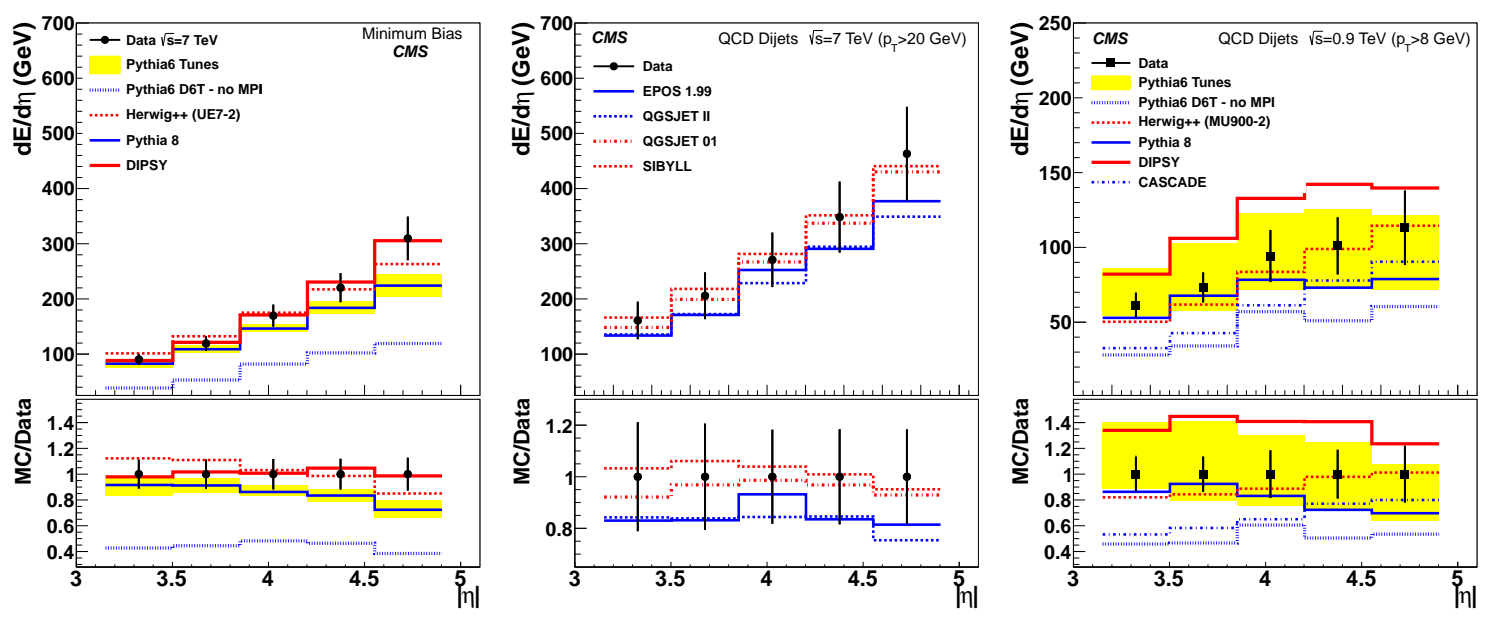

Figure 1: Energy flow in the forward region for minimum bias event and events containing a central dijet system.

surement of the energy flow in minimum bias events at $\sqrt{s}=7 \mathrm{TeV}$ is compared to predictions of different Monte Carlo event generators (MC) used to describe $p p$ physics. The prediction without including multiparton interactions is significantly below the measurement. Including multi-parton interactions improves the description of the measurement. The band shows a spread of different tunes of the PYTHIA6 [3] Monte Carlo event generator. HERWIG [5, 6] with special tunes for the two center of mass energies comes close to the measurement, whereas PYTHIA8[4] lies below the measurement at $\sqrt{s}=7 \mathrm{TeV}$. In Fig. $1 \mathrm{~b}$ it is shown that the data are well described by MC generators used in the simulation of cosmic air showers. The observed rise of energy flow with increasing $|\eta|$ corresponds to a approximately constant transverse energy flow.

In Fig. 1c the measurement of the energy flow in events containing a dijet system with $E_{t}>$ $20 \mathrm{GeV}$ in $|\eta|<2$ is compared to predictions of MC generators. Predictions without multiparton interactions (PYTHIA as well as CASCADE [7, 8]) are much below the measurements, while predictions using multiparton interactions are close to the data. The spread of predictions obtained with different tunes shown as the band in Fig. 1c illustrates the sensitivity to the tuning parameters for multiparton interaction. The energy flow in dijet events corresponds to an $E_{T}$ flow, which decreases with increasing $|\eta|$.

The energy distribution in the range $3.0<|\eta|<4.9$ in events where a $W$-boson is detected via a lepton with $p_{t}>25 \mathrm{GeV}$ in the range $|\eta|<2.5$ is measured [9]. The energy is obtained from the 

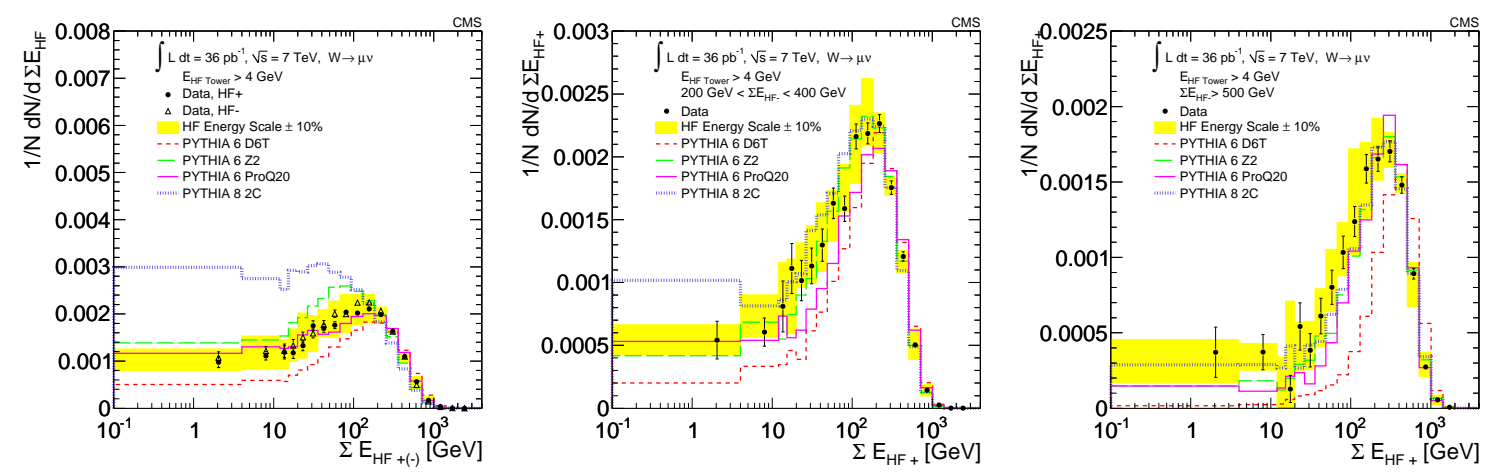

Figure 2: Energy distribution in the range $3<\eta<4.9$ as a function of the energy deposit in the range $-4.9<\eta<-3$.

sum of individual calorimeter towers of the hadronic forward (HF) calorimeter with a minimum energy threshold of $4 \mathrm{GeV}$. The energy scale uncertainty in the calorimeter is estimated to be about $\pm 10 \%$. The energy distributions obtained from the calorimeters on both sides of the CMS detector $(\mathrm{HF} \pm)$ are identical.

In Fig. 2 the forward energy in $\mathrm{HF}+$ is shown for events with a central $W$ and energy deposits in HF- between 20-100 GeV (low), 200-400 GeV (medium) and above $500 \mathrm{GeV}$ (high). In the medium energy interval the differences between the data and the different tunes are smaller than seen in the case of the inclusive energy distributions (see Fig.2 in [9]). In the low energy interval the measured energy distribution is badly modeled by all MC tunes. When selecting events with high energy deposits we find that PYTHIA 8 provides a rather good description of the energy. In contrast to that, all other tunes predict more events with large HF+ energy than observed in data.

\section{Forward jet cross sections}

The cross section of jets with $p_{t}>35 \mathrm{GeV}$ reconstructed with the anti- $k_{t}$ jet algorithm has been measured in the range $3.2<|\eta|<4.7$ (Fig. 3 left) [11]. In a separate measurement an additional jet with $p_{t}>35 \mathrm{GeV}$ in the central region with $|\eta|<2.8$ has been required Fig.3 (middle and right). In all measurements the dominant systematic uncertainty is the jet energy scale. While the forward jet cross section (Fig. 3 left) is rather well described by all different predictions, the associated central jet cross section (Fig. 3, middle and right) is not well described by PYTHIA, POWHEG+PYTHIA [12] and CASCADE, while a reasonable description is obtained with HERWIG, POWHEG+HERWIG and HEJ [13, 14].

\section{Conclusion}

The forward energy flow in minimum bias events and in events with a central dijet of a central $W$ boson has been measured. The MC models which describe event properties in the central region are not able to describe all features of the forward energy flow measurements.

The forward jet production cross section is reasonably well described by different theoretical predictions. The central jet cross section associated with a forward jet provides additional constraints to the theoretical modelling of multiparton production with large rapidity separations. 

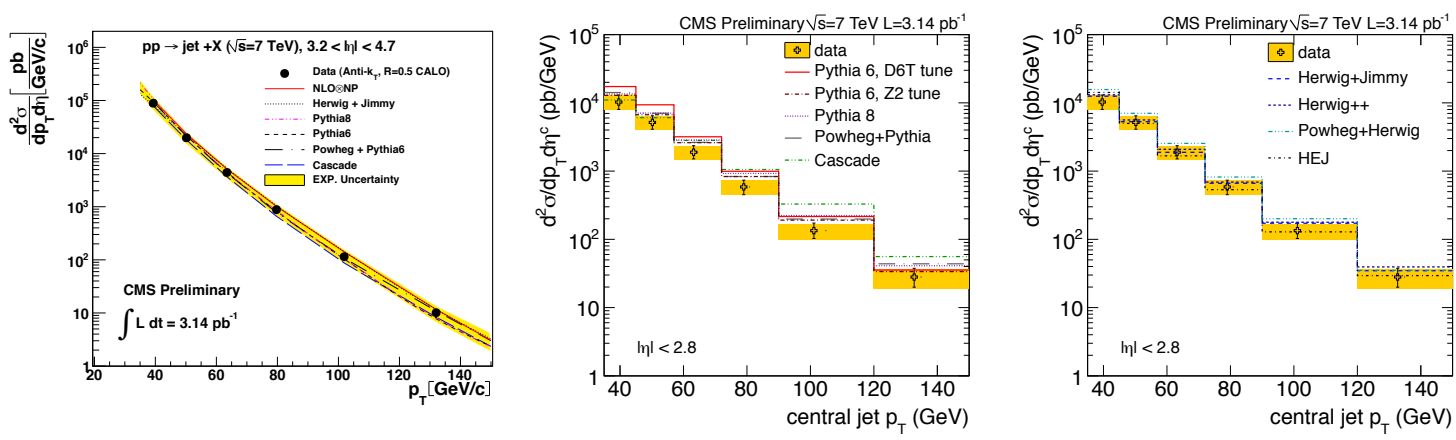

Figure 3: Cross section of forward jets $(3.2<|\eta|<4.7$, left $)$ and associated central jets $(|\eta|<2.8$ middle and right).

\section{References}

[1] CMS Collaboration, The CMS experiment at the CERN LHC. JINST, 3:S08004, 2008.

[2] CMS Collaboration, "Measurement of energy flow at large pseudorapidities in pp collisions at $\sqrt{s}=$ 0.9 and $7 \mathrm{TeV}$," arXiv:1110.0211 [hep-ex].

[3] T. Sjostrand, S. Mrenna, and P. Skands, "PYTHIA 6.4 Physics and Manual", JHEP 05 (2006) 026,

[4] T. Sjostrand, S. Mrenna, and P. Z. Skands, "A Brief Introduction to PYTHIA 8.1", Comput.Phys.Commun. 178 (2008) 852-867,

[5] G. Marchesini et al., "HERWIG: A Monte Carlo event generator for simulating hadron emission reactions with interfering gluons. Version 5.1", Comput. Phys. Commun. 67 (1992) 465-508.

[6] M. Bahr et al., "Herwig++ 2.2 Release Note", arXiv:0804.3053.

[7] H. Jung and G. P. Salam, "Hadronic final state predictions from CCFM: The hadron- level Monte Carlo generator CASCADE", Eur. Phys. J. C19 (2001) 351-360,

[8] H. Jung, S. Baranov, M. Deak et al., "The CCFM Monte Carlo generator CASCADE 2.2.0", The European Physical Journal C - Particles and Fields 79 (2010) 1237-1249,

[9] CMS Collaboration, "Forward Energy Flow, Central Charged-Particle Multiplicities, and Pseudorapidity Gaps in W and Z Boson Events from pp Collisions at $7 \mathrm{TeV}$," arXiv:1110.0181 [hep-ex].

[10] CMS Collaboration. Measurement of forward jets in proton-proton collisions. CMS Physics Analysis Summary CMS-PAS-FWD-10-003, 2010.

[11] CMS Collaboration. Cross section measurement for simultaneous production of a central and a forward jet in proton-proton collisions at $\sqrt{s}=7 \mathrm{TeV}$. CMS Physics Analysis Summary CMS-PAS-FWD-10-006, 2010.

[12] S. Frixione, P. Nason and C. Oleari, "Matching NLO QCD computations with Parton Shower simulations: the POWHEG method", JHEP 11 (2007) 070,

[13] J. R. Andersen, J. M. Smillie, "Constructing All-Order Corrections to Multi-Jet Rates", JHEP 1001:039 (2010).

[14] J. R. Andersen, J. M. Smillie, "Multiple Jets at the LHC with High Energy Jets", arXiv:1101.5394 (2010). 